\title{
Applying ESPGHAN and WGO guidelines of celiac disease on Iraqi patients
}

\author{
Alaa S. Alattabi and Israa H. Aljerrah ${ }^{a^{*}}$
}

aDepartment of Microbiology, College of Medicine, University of Karbala, Karbala, Iraq.

*Correspondence to Israa H. Aljerrah (email: israa4019@gmail.com).

(Submitted: 03 Auqust 2018 - Revised version received: 07 September 2018 - Accepted: 18 October 2018 - Published online: 26 December 2018)

\begin{abstract}
Objective The goal of this study is to check the degree to which the physicians and pediatricians can apply European Society for Paediatric Gastroenterology, Hepatology and Nutrition (ESPGHAN) and World Gastroenterology Organization (WGO) criteria of celiac disease (CD) on their patients in Karbala city in Iraq.

Methods This is a cross-sectional study conducted in Karbala city hospitals (Al-Hussein Medical City Hospital, The Pediatric Teaching Hospital) for the period from August 2017 to February 2018. A random selection of 108 CD patients was performed and the clinical data including the biopsy results were collected directly from patients or their family members through a questionnaire sheets. Statistically, the quantitative variables were analyzed using non-parametric $t$-test and the qualitative variables were analyzed using Chi-square test.

Results The study shows that of the total 108 patients, only $n=43$ (39.8\%) of candidates who did biopsy even with tissue TG (tTG) IgA $>10$ fold upper limit of normal (ULN) (200 RU/ml and more) due to the shortage in endomysial antibody (EMA)/HLA tests recommended by ASPGHAN to omit duodenal biopsy, while $n=51$ (47.2\%) accomplished only one of the triple tests (only tTG IgA >10-fold ULN) suggested by ASPGHAN and then applied challenge test and $n=14$ (12.9\%) achieved none of the triple tests (tTG IgA antibody titer $>5$-fold ULN, but $<10$-fold ULN). On the other hand, the WGO guideline is more suitable if properly applied as it considers tTG (IgA) a convenient substituent of EMA test where high tTG (IgA) serum level being diagnostic and to be confirmed by the available DGP (IgA and IgG) antibodies.

Conclusion The WGO guideline for symptomatic and asymptomatic patients is more applicable for the areas with limited facilities, while ESPGHAN guideline for pediatrics can be applied to a less degree. Generally in Iraq there is a delay in disease diagnosis due to many reasons to be evaluated.

Keywords celiac disease, ESPGHAN criteria, WGO guideline, tTG (IgA) antibodies, challenge test, biopsy findings
\end{abstract}

\section{Introduction}

Celiac disease $(\mathrm{CD})$ is a chronic systemic immune-mediated disorder associated with variable small-intestinal mucosal lesion caused by gluten consumption in genetically susceptible individuals. ${ }^{1,2} \mathrm{CD}$ prevalence is about $1 \%$ of the general population. $^{3}$ The continuous 'Westernization' of the diet ${ }^{4,5}$ may account for CD being also common in many developing countries, in particular the Middle East.

Nowadays, in particular resource-rich countries, more heterogeneous clinical manifestations of CD may be reported, including typical gastroenterological symptoms as well as a myriad of extra-intestinal disorders. ${ }^{7}$ The GI symptoms (diarrhea and malabsorption syndrome) are predominating in young children, while failure to thrive, short stature, and other growth problems occur in all pediatric age groups. ${ }^{8}$ Generally extra-intestinal (atypical) clinical features occur in children more than 5-year-old and so in adults. ${ }^{9}$ Diagnostic approach in CD includes: clinical manifestations, serological tests, genetic study (HLA-DQ2/DQ8 typing) and small intestinal biopsy histological findings (Husby et al., 2013). Serum tissue TG (tTG) (IgA) measurement is the premier testing recommended for individuals due to its excellent standardization and being a high sensitive and specific test. ${ }^{10,11}$

The guidelines of the European Society for Paediatric Gastroenterology, Hepatology and Nutrition (ESPGHAN) authorize for $\mathrm{CD}$ diagnosis without biopsies in children present a distinctive intestinal clinical features and levels of TG2-IgA antibodies 10-fold or more than the upper normal level (UNL), detection of anti-endomysial antibody in another test plus genetic study (HLA-DQ2/DQ8 typing) for confirmation [triple test ESPGHAN criteria], while proposes shifting to endoscopy and biopsy if endomysial antibody (EMA) test and HLA-typing are not available.

Recent guidelines from the World Gastroenterology Organization (WGO) for diagnosis of CD take in consideration the resources available in each country. According to WGO, the experts propose that the primary test for CD patients with or without symptoms is the measurement of anti-tTG (IgA) and total IgA serum levels with recommendation for adding another test either to confirm the first test positivity or to support the low (borderline) anti-tTG (IgA). These tests include EMA or DGP antibodies where in complaining patients, the guideline showed that the DGP (IgA and $\operatorname{IgG}$ ) have similar performance to tTG (IgA) antibodies.

The combined tests of DGP (IgG) and tTG (IgA) are of particular benefit as beside detection of IgA-deficient CD patients, IgG-DGP can also detect small percent of patients who are normal for IgA but are negative for IgA-TG2 tests. Another point is that some data demonstrate that using these two tests directing toward different antigens to be more useful than using tests act on the same autoantigen like tTG (IgA) and EMA (IgA). ${ }^{12} \mathrm{~A}$ recent study showed that a simultaneous testing of tTG (IgA) and DGP (IgG) antibodies constitutes a potentially accurate diagnostic tool for $\mathrm{CD} \cdot{ }^{13}$ However, a tTG (IgA) positivity and clinical response on adherence to a GFD might be enough to consider CD diagnosis in countries with limited facilities. ${ }^{12,14}$

At the time, WGO necessitates small intestinal biopsy in certain conditions when patients not present the malabsorptive manifestations or when serological tests either low or negative, the WGO also propose omission of intestinal biopsy in other conditions where positive and negative points of $\mathrm{CD}$ can be 
discussed with well-experienced specialists. This is convenient in areas where healthcare facilities are restricted. ${ }^{12}$ Gluten challenge is the mode by which a clinically suspected patient, but uncertain $\mathrm{CD}$ where formerly managed by gluten restriction returns to a usual, gluten-containing diet, along medical monitoring, enabling the disease diagnosis. ${ }^{15,16}$ In the past this test was routinely used to diagnose $\mathrm{CD}$, however a less frequent use nowadays due to the high accuracy of serological tests.

The study aims to verify the degree to which we can apply these guidelines on CD diagnosis at the level of Karbala city.

\section{Materials and Methods}

Ethically, the selection of the study groups and data collection were accomplished after we have been taken the approval of Karbala Health Director to work in its hospitals and a practical supervisor has been set in each hospital to follow the workflow.

A cross-sectional study was carried out for the period from August 2017 to February 2018 in Karbala province hospitals. A total of 108 patients (old and new cases, pediatric and adult age groups) who were attending endocrine centers in Al-Hussein Medical City Hospital and The Pediatric Teaching Hospital were randomly selected and enrolled in the study according to inclusion and exclusion criteria. The age groups ranging between 1 and 58 years with 68 of them are below 18 years. The patient's sex was 75 female vs 33 male.

Clinical data includes: age, sex, residence, complaints, the period of clinical presentation, duration of disease (old cases), family history, skin manifestations, associated autoimmune disorders, serum antibody titer, indications for endoscopy and biopsy result, challenge test, treatment, complications, and more others collected directly from patients or their family members through a questionnaire sheets. The serological markers which currently used as a first step in the screening for CD (tTG IgA, IgG and DGP IgA, IgG) are measured by antibody sandwich enzyme-linked immunosorbant assay (ELISA) technique which was carried out by the laboratory staff of Al-Hussein Medical City Hospital and The Pediatric Teaching Hospital where both antibodies are available in Al-Hussein Medical City lab and only tTG (IgA, IgG) is available in Pediatric Teaching Hospital. The EUROIMMUN Medizinische Labordiagnostika AG assay provides a semi-quantitative or quantitative in vitro assay for human antibodies in serum or plasma.

Data analysis was performed using the SPSS version 18 software package (Statistical Package for Social Science; Inc., Chicago, IL, USA). Quantitative data were expressed as mean \pm standard deviation (SD), and analyzed using non-parametric $t$-test. On the other hand, qualitative data were expressed as numbers $(N)$ and percentages, and analyzed using Chi-square test. Pearson correlation analysis was used to reveal the association between two or more of the related quantitative variables.

${ }^{*}$ A $P$-values $<0.05$ were considered as statistically significant.

${ }^{*}$ Permission of patients to publish the study.

\section{Results}

\section{Socio-demographic Characteristics of Celiac Patients}

Children account to about two third $(n=68 ; 63 \%)$ of the celiac patients, while females in this study group are more than males (69.4\% vs $30.6 \%)$ with the female to male ratio being $2.27: 1$ and about three quarters of patients are of urban residence ( $n=80 ; 74.1 \%$ vs $n=28 ; 25.9 \%)$.

Most of patients revealed typical or intestinal clinical features, while atypical or extra-intestinal presentation account for small percentage ( $83.8 \%$ vs $16.2 \%)$. Another point is that the majority of patients were complaining for at least few months to years before being diagnosed to have CD.

\begin{tabular}{lcc}
\hline \multicolumn{3}{l}{$\begin{array}{l}\text { Table 1. Socio-demographic characteristics of celiac patients. } \\
\text { The values are in percentage and numbers }\end{array}$} \\
\begin{tabular}{lcc}
\hline Age (years) & Number & Percentage (\%) \\
\hline$<18$ & 68 & 63 \\
18 and above & 40 & 37 \\
Total & 108 & 100 \\
Mean \pm SD & $18.39 \pm 14.08$ & \\
Range & $1-58$ & \\
\hline Gender & Number & Percentage (\%) \\
\hline Male & 33 & 30.6 \\
Female & 75 & 69.4 \\
Total & 108 & 100 \\
\hline Residence & Number & Percentage (\%) \\
\hline Urban & 80 & 74.1 \\
Rural & 28 & 25.9 \\
Total & 108 & 100 \\
\hline
\end{tabular}
\end{tabular}

$\begin{aligned} & \text { Table 2. The clinical presentations of celiac patients. The } \\
& \text { values are in percentage and numbers }\end{aligned}$
\begin{tabular}{lcc}
\hline Clinical presentation & Number & Percentage (\%) \\
\hline Intestinal & 88 & 83.8 \\
Extra-intestinal & 17 & 16.2 \\
Total & 105 & 100 \\
\hline Period of presentation & Number & Percentage (\%) \\
\hline Months & 48 & 45.7 \\
Years & 57 & 54.3 \\
Total & 105 & 100 \\
\hline
\end{tabular}

Tissue TG IgA 10 fold the ULN and more Tissue TG IgAless than 10 fold the ULN

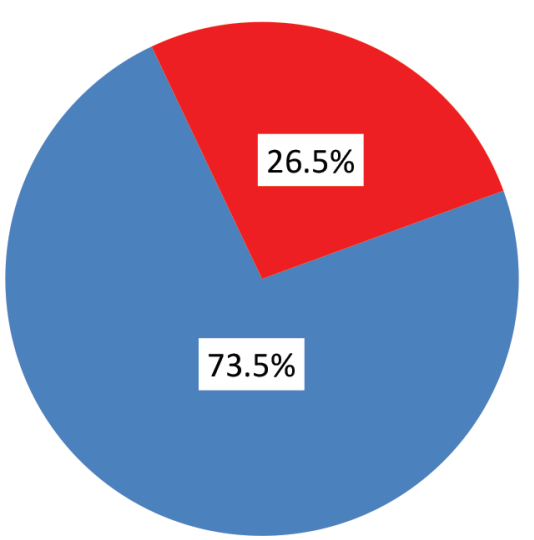

Fig. 1 Percentage of tTG IgA titer of CD patients. 


\section{Serological Markers of CD}

The candidates also revealed that the percentage of the 10 -folds the upper limit of normal (ULN) range of the antibody tTG IgA (cut-off), i.e. $200 \mathrm{RU} / \mathrm{ml}$ and more, was positive in about three quarters of the patients.

Notably, Fig. 2 shows that there was no significant association of (difference in) tTG IgA titer between age groups $(P=0.6)$.

\section{Small Intestine Biopsy Results}

A small intestine endoscopy and biopsy had been done for all adult age group (40) and only three children and the results according to modified Marsh ${ }^{17}-$ Oberhuber $^{18}$ classification demonstrates that M2 accounts for about half of cases as shown in Fig. 3.

The triple tests suggested by ASPGHAN to omit biopsy in symptomatic patients include TG2-IgA antibodies

$$
\text { 를 High Tissue TG } \mid g A>200 \mathrm{RU} / \mathrm{ml}
$$

n Tissue TG IgA below 200RU/m

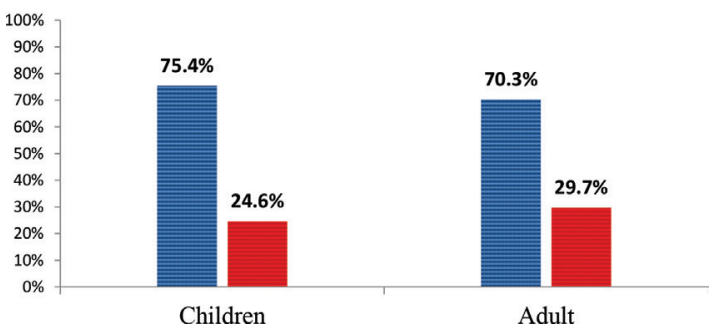

\section{Fig. 2 Association of tTG IgA titer with age groups.}

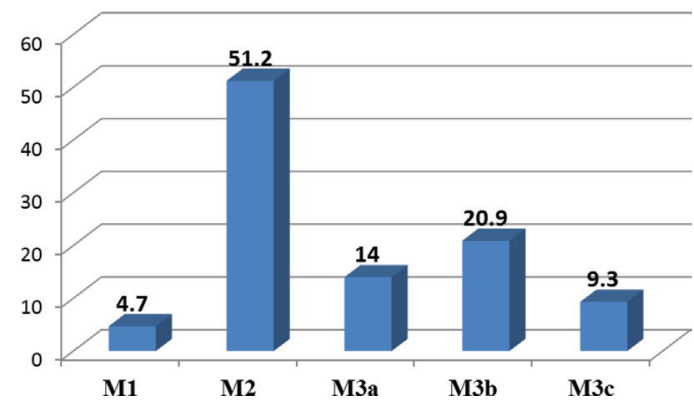

Fig. 3 Small intestine biopsy results according to modified Marsh-Oberhuber classification. 10-fold or more than the UNL, detection of anti-endomysial antibody in another test plus genetic study (HLA-DQ2/ DQ8 typing) for confirmation. ${ }^{1}$ Table 4 shows that of the total 108 patients, only $n=43(39.8 \%)$ of candidates who did biopsy even with tTG IgA >10-fold ULN (200 RU/ml and more) due to the shortage in EMA/HLA tests, while $n=51(47.2 \%)$ accomplished one of the triple tests (only tTG IgA >10-fold ULN) suggested by ASPGHAN and then applied challenge test, while $n=14(12.9 \%)$ who have tTG IgA $>5$-fold ULN, but $<10$-fold ULN and also passed to challenge test. Nevertheless, the WGO guideline can be applied in all the patients $(n=108 ; 100 \%)$ as high tTG IgA titer is revealed in about three quarter of the patients and the already available DGP (IgA, IgG) antibodies to confirm positivity.

\section{Correlation of tTG (IgA) Level with small Intestine Biopsy Results}

Regarding the biopsy results, patients who had undergone small intestine endoscopy and biopsy were divided into two categories: those with M3 (a-c) grade of modified MarshOberhuber classification show mean autoantibody level more than that those with M2 grade, however, this difference not reached significance ( $P$-value 0.07$)$.

\section{Discussion}

According to the clinical data collected and analyzed, the study demonstrated that children account to about two third ( $n=68 ; 63 \%$ ) of the celiac patients, a result consistent with the results of other previous studies which showed that $64 \%$ of patients with CD in Karbala were among age group of 2-10 years. ${ }^{19}$ The females are more affected than males $(n=75$; $69.4 \%$ vs $n=33 ; 30.6 \%)$ with the female to male ratio being

Table 5. Correlation of tTG (IgA) antibody titers with biopsy findings

\begin{tabular}{llllll}
\hline Biopsy findings & & No. & Mean & SD & P-value $^{*}$ \\
\hline \multirow{3}{*}{ tTG (IgA) } & M2 & 22 & 252.56 & 131.07 & 0.07 \\
& M3 & 19 & 337.47 & 153.87 & \\
& $(\mathrm{a}-\mathrm{c})$ & & & & \\
\hline
\end{tabular}

*Pearson correlation was used. Correlation is significant at $P$-value of 0.05 .

\begin{tabular}{|c|c|c|c|c|c|c|}
\hline \multirow{2}{*}{ ASPGHAN triple test application } & \multirow{2}{*}{ Number } & \multirow{2}{*}{$\begin{array}{c}\text { Percentage } \\
(\%)\end{array}$} & \multicolumn{2}{|c|}{ Al-Hussein Medical City } & \multicolumn{2}{|c|}{ Pediatric Teaching Hospital } \\
\hline & & & Number & Percentage (\%) & Number & Percentage (\%) \\
\hline Only tTG IgA > 10-fold ULN (1/3) & 51 & 47.3 & 0 & 0 & 51 & 75 \\
\hline tTG IgA >10-fold ULN + EMA (2/3) & 0 & 0 & 0 & 0 & 0 & 0 \\
\hline tTG IgA >10-fold ULN + HLA typing (2/3) & 0 & 0 & 0 & 0 & 0 & 0 \\
\hline tTG IgA >10-fold ULN + EMA + HLA typing (3/3) & 0 & 0 & 0 & 0 & 0 & 0 \\
\hline $\begin{array}{l}\text { Biopsy if EMA/HLA tests not available or tTG IgA } \\
<10 \text {-fold ULN }\end{array}$ & 43 & 39.8 & 40 & 100 & 3 & 4.4 \\
\hline None of the triple test & 14 & 12.9 & 0 & 0 & 14 & 20.6 \\
\hline Total & 108 & 100 & 40 & 100 & 68 & 100 \\
\hline \multirow{2}{*}{ WGO guideline application } & \multirow{2}{*}{ Number } & \multirow{2}{*}{$\begin{array}{c}\text { Percentage } \\
(\%)\end{array}$} & \multicolumn{2}{|c|}{ Al-Hussein Medical City } & \multicolumn{2}{|c|}{ Pediatric Teaching Hospital } \\
\hline & & & \multicolumn{2}{|c|}{ Number } & \multicolumn{2}{|c|}{ Number } \\
\hline High tTG IgA titer & 108 & 100 & \multicolumn{2}{|c|}{40} & \multicolumn{2}{|c|}{68} \\
\hline DGP (lgA/lgG) for confirmation & 0 & 0 & \multicolumn{2}{|r|}{0} & \multicolumn{2}{|r|}{0} \\
\hline Total & 108 & 100 & \multicolumn{2}{|r|}{40} & \multicolumn{2}{|r|}{68} \\
\hline
\end{tabular}


2.27:1, a results which usually observed as there is a higher rate of autoimmune disease among females. ${ }^{20}$ Most of the patients (adults $90 \%$ and children $80 \%$ ) revealed typical or intestinal clinical features, while atypical or extra-intestinal presentation account for small percentage ( $n=88 ; 83.8 \%$ vs $n=17 ; 16.2 \%)$ and only three patients of total had silent presentation and diagnosed during follow-up tests for DM type 1. Although atypical (non-classical) manifestations are more in western patients, ${ }^{12}$ however, the predominance of intestinal (typical) manifestations in both age groups of candidates may not reflect the real situation as many undiagnosed CD patients missed among clinics of different specialties searching for symptomatic management of their complaints where low awareness about the extra-intestinal (atypical) manifestations by some clinicians may also contribute to this result.

Given the increasing rate in $\mathrm{CD}$ diagnosis all over the world, many areas including Iraq do not always have the sophisticated techniques required for disease diagnosis like anti-EMA and genetic study testing.

When reviewing the guidelines of the ESPGHAN which allow CD diagnosis without biopsies in children with obvious symptoms and levels of (TGA-IgA) antibodies 10-fold or more the ULN, with positive EMA in a different blood sample as a confirmatory test and positive HLA-DQ2/DQ8 typing [triple test ESPGHAN criteria]. ${ }^{1}$ To verify the study candidates with ESPGHAN approach for diagnosis, and although about three quarters of the patients have high serum tTG IgA antibody titer [ 10 -folds the ULN range] of this antibody cut-off (i.e. 200 $\mathrm{RU} / \mathrm{ml}$ and more), we have to explain some points:

1. There is no genetic study (HLA-DQ2/DQ8 typing) at least at the level of Kabala province to document the positivity of HLA-typing.

2. Anti-EMA assay is also not available in Kabala province.

Nevertheless these points may not represent the stumbling block for the diagnosis as many recent data revealed the possibility to overcome these points, of which Werkstetter et al. ${ }^{21}$ in a prospective study to validate ESPGHAN approach on children who complaining of malabsorption symptoms instead of any symptom show that the inclusion of HLA analyses did not increase accuracy of diagnosis. Also Mubarak et al. ${ }^{22}$ concluded that pediatric patients with a tTGA level $\geq 100$ $\mathrm{U} / \mathrm{mL}$ in whom symptoms improve upon consuming a GFD may not need a small intestinal biopsy to confirm CD.

Mubarak et al. ${ }^{23}$ in another study based on prospective data confirms omitting of a small intestinal biopsy for the diagnosis of CD in symptomatic patients with tTG IgA serum level $\geq 100 \mathrm{U} / \mathrm{mL}$. The gastroenterologists in the endocrine center of the AI-Hussein Medical City Hospital when serology result reveals high tTG (IgA) antibodies, they consider a small intestine biopsy is a must for diagnosis of CD in adults [biopsy done for $n=43$ (40 adults and three children); 39.8\%]. On the other hand, pediatricians shift to challenge test (not biopsy) which is regarded as a confirmatory test for children with clinical manifestations of CD and a highly positive tTG (IgA) level and their parents refuse a small intestine endoscopy and biopsy as invasive procedure for diagnosis. Of note all the pediatric patients $(n=65 ; 60.2 \%)$ were positive for challenge test. Hence very high levels of serum anti-tTG IgA might be enough on the basis of suggestive intestinal clinical manifestations demonstrated in most of the patients, a point supported by the
WGO diagnostic protocol, especially in regions with limited resources (shortage of sophisticated techniques), considered tTG as an acceptable proxy of the EMA antibodies ("The EMA test needs expert observers, and detection of tTG antibodies by ELISA tests should therefore be recommended in settings with low expertise") [11]. The WGO also recommended the evaluation of the local resources available to develop a diagnostic protocol. ${ }^{24}$ and his team in a population-based study found that serum level of tTG (IgA) can identify celiac disease patients with about $90 \%$ PPVs. Another supporting point for WGO guideline is the availability of antibodies to DGP (IgA and $\operatorname{IgG}$ ), which are in complaining patients having similar performance to tTG (IgA) antibodies. However an important fact should be mentioned that DGP (IgA and IgG) antibodies in the main labs of related hospitals are completely neglected by many gastroenterologists and pediatricians and they think that it is the old low-accurate anti-gliadin antibody test, so they sent only for tTG (IgA) antibodies, while others believe that tTG (IgA) antibodies are enough for CD serology. This implies that inspite of the specialists ability to apply WGO guideline in most of the patients, but improper application of this guide may occur when some clinicians neglect this test in the absence of EMA test.

Measurement of antibodies serum levels was carried out using quantitative data for caliculation, implying more accuracy for diagnosis in contrast to some laboratories that produce semi-quantitative data, making the assessment uncertain. ${ }^{25}$

When correlating tTG (IgA) level with small intestine biopsy results, we found that patients with M3 $(\mathrm{a}-\mathrm{c})$ grade of modified Marsh-Oberhuber classification have mean autoantibody levels $(337.47 \mathrm{RU} / \mathrm{ml})$ more than that those with M2 grade (mean tTG (IgA) level $252.56 \mathrm{RU} / \mathrm{ml}$ ), however, this difference not reached significance ( $P$-value 0.07$)$, an information that meriting further evaluation, where small sample size might be a confounding factor. Generally these findings goes with Hawamdeh et al. ${ }^{26}$ findings in 2016 who concluded a significant association between the degree of duodenal damage and anti-tTG titers, where anti-tTG titer more than 10 -folds the ULN $(\geq 180 \mathrm{U} / \mathrm{mL})$ was significantly associated with Marsh III enteropathy; this strong association of high anti-tTG titer and severity of intestinal damage might provide a remarkable evidence for diagnosis of $\mathrm{CD}$ when endoscopy is not applicable and fortified by positive EMA test or HLA typing.

Another points deserves discussion are:

A- The prolonged period of presentation noticed in the majority of patients before diagnosis which may be attributed to many causes including: 1 . Limited resources of sophisticated techniques important to confirm diagnosis. 2. Lacking of screening tests in the primary health centers which are essential to detect many complainers as most people attend these centers for the first time.

Of note, only the endocrine center in The Pediatric Teaching Hospital carries out screening tests for children with positive family history for CD or children with other autoimmune diseases mostly type $1 \mathrm{DM}$. 3. Celiac disease is the last in the list of differential diagnosis made for patients by most specialists, where irritable bowel, inflammatory bowel disease, Giardiasis, and Helicobacter pylori infection are in the top of the list. 4. Patients jumping from one specialist to another seeking for response while others refuse biopsy at the 
beginning of disease and so wasting more time before diagnosis being confirmed. 5 . Lacking of education about the disease, environmental factors and motivation toward life-long restriction of dietary gluten as most patients think that $\mathrm{CD}$ is transient rather than permanent disorder.

B- All candidates had started a GFD, but unfortunately inadequate adherence noticed in most of the patients, however, some clinical and serological responses had been shown in many of them.

All these points are to be considered in the future which may help in applying ESPGHAN and WGO guidelines to uncover much of the underdiagnosed cases in Iraq.

\section{Conclusion}

The WGO guideline is more suitable if properly applied for the Iraqi patients due to shortage of sophisticated techniques, while ESPGHAN guideline for pediatrics can be applied to a less degree. Generally here in Iraq there is an obvious delay in disease diagnosis due to many reasons to be evaluated.

\section{Conflict of Interest}

None.

\section{References}

1. Husby S, Koletzko S, Korponay-Szabo IR et al. European Society for Pediatric Gastroenterology, Hepatology, and Nutrition guidelines for the diagnosis of coeliac disease. J Pediatr Gastroenterol Nutr. 2012;54:136-160.

2. Ludvigsson JF, Leffler DA, Bai JC, Biagi F, Fasano A, Green PH, et al. The Oslo definitions for coeliac disease and related terms. Gut. 2013;62:43-52.

3. Rubio-Tapia A, Hill ID, Kelly CP, Calderwood AH, Murray JA; American College of Gastroenterology. ACG clinical guidelines: diagnosis and management of celiac disease. Am J Gastroenterol. 2013;108:656-676.

4. Fasano A, Catassi C. Celiac disease. N Engl J Med. 2012;367:2419-2426.

5. Sapone A, Bai JC, Ciacci C, Dolinsek J, Green PH, Hadjivassiliou M, et al. Spectrum of gluten-related disorders: consensus on new nomenclature and classification. BMC Med. 2012:10:13.

6. Dalgic B1, Sari S, Basturk B, Ensari A, Egritas O, Bukulmez A, et al. Prevalence of celiac disease in healthy Turkish school children. Am J Gastroenterol. 2011;106:1512-1517.

7. Jeon MK, Klaus C, Kaemmerer E, Gassler N. Intestinal barrier: molecular pathways and modifiers. World J Gastrointest Pathophysiol. 2013:4:94-99.

8. Reilly NR, Aguilar K, Hassid BG, Cheng J, Defelice AR, Kazlow P, et al. Celiac disease in normal-weight and overweight children: clinical features and growth outcomes following a gluten-free diet. J Pediatr Gastroenterol Nutr. 2011;53:528-31.

9. Akirov A, Pinhas-Hamiel O. Co-occurrence of type 1 diabetes mellitus and celiac disease. World J Diabetes. 2015;6:707-714.

10. Zintzaras E, Germenis AE. Performance of antibodies against tissue transglutaminase for the diagnosis of celiac disease: meta-analysis. Clin Vaccine Immunol. 2006;3:187-192.

11. Lewis NR, Scott BB. Meta-analysis: deamidated gliadin peptide antibody and tissue transglutaminase antibody compared as screening tests for coeliac disease. Aliment Pharmacol Ther. 2010;31:73-81.

12. Bai JC, Ciacci C, Corraza GR, Fried M, Olano C, Rostami-Nejad M, et al. World Gastroenterology Organisation Practice Guidelines: Celiac Disease. World Gastroenterology Organisation, Milwaukee, WI, USA, Vol. 48, 2013, pp. 1-18.

13. Hong YY. Deaminated gliadin peptide antibody in the diagnosis of celiac disease. Int J Celiac Dis. 2015;3:56-57.

14. Kelly CP, Bai JC, Liu E, Leffler DA. Advances in diagnosis and management of celiac disease. Gastroenterology. 2015;148:1175-1186.
15. Lahdeaho ML, Mäki M, Laurila K, Huhtala H, Kaukinen K. Small-bowel mucosal changes and antibody responses after low-and moderatedose gluten challenge in celiac disease. BMC Gastroenterol. 2011; $11: 129$.

16. Leffler, D. Schuppan, D. Pallav, K. Najarian, R. Goldsmith, JD, Hansen, J, et al. Kinetics of the histological, serological and symptomatic responses to gluten challenge in adults with coeliac disease. Gut. 2012;62:996-1004.

17. Marsh MN. Mucosal pathology in gluten sensitivity. In: Marsh MN, Ed. Coeliac Disease, Blackwell Science Ltd., Oxford, UK, 1992;136-191.

18. Oberhuber G. Histopathology of celiac disease. Biomed Pharmacother. 2000;54:368-372

19. Hameed WS, Abdul-Mehdi RJ, Tarish HR, Alsherees HAA. Prevalence of DQ2, DQ8 and DR4 Alleles in Iraqi Celiac Patients. Int Arch BioMed Clin Res. 2016;2:118-121.

20. Dixit R, Lebwohl B, Ludvigsson JF, Lewis SK, Rizkalla-Reilly N, Green PH Celiac disease is diagnosed less frequently in young adult males. Dig Dis Sci. 2014:59:1509-1512.

21. Werkstetter KJ, Korponay-Szabó IR, Popp A, Villanacci V, Salemme M, Heilig $\mathrm{G}$, et al. Accuracy in diagnosis of celiac disease without biopsies in clinical practice. Gastroenterology. 2017;153:924-935.

22. Mubarak A, Wolters VM, Gerritsen SA, Gmelig-Meyling FH, Ten Kate FJ, Houwen RH. A biopsy is not always necessary to diagnose celiac disease. J Pediatr Gastroenterol Nutr. 2011;52:554-557.

23. Mubarak A, Wolters VM, Gmelig-Meyling FH, Ten Kate FJ, Houwen RH. Tissue transglutaminase levels above $100 \mathrm{U} / \mathrm{mL}$ and celiac disease: a prospective study. World J Gastroenterol. 2012;18:4399-4403.

24. Ermarth A, Bryce M, Woodward S, Stoddard G, Book L, Jensen MK. Identification of pediatric patients with celiac disease based on serology and a classification and regression tree analysis. Clin Gastroenterol Hepatol. 2017;15:396-402.e2.

25. Tucci F, Astarita L, Abkari A, Abu-Zekry M, Attard T, Ben Hariz M, et al. Celiac disease in the Mediterranean area. BMC Gastroenterol. 2014;14:24.

26. Hawamdeh H, Al-Zoubi B, Al Sharqi Y, Qasrawi A, Abdelaziz Y, Barbar M. Association of tissue transglutaminase antibody titer with duodenal histological changes in children with celiac disease. Gastroenterol Res Pract. 2016;2016:6718590 